\title{
Gutzwiller Approximation in Degenerate Hubbard Models
}

\author{
Jian Ping Lu \\ Department of Physics and Astronomy \\ University of North Carolina at Chapel Hill \\ Chapel Hill, North Carolina 27599 \\ jpl@physics.unc.edu
}

(July 28, 2018)

\begin{abstract}
Degenerate Hubbard models are studied using the Generalized-GutzwillerApproximation. It is found that the metal-insulator transition occurs at a finite correlation $U_{c}$ when the average number of electrons per lattice site is an integer. The critical $U_{c}$ depends sensitively on both the band degeneracy $N$ and the filling $x$. A derivation is presented for the general expression of $U_{c}(x, N)$ which reproduces all previously known Gutzwiller solutions, including that of the Boson Hubbard model. Effects of the lattice structure on the metal-insulator transition and the effective mass are discussed.
\end{abstract}

PACS numbers: 71.10.+x,71.30.+h,74.70.W

Typeset using REVTEX 
The simple Hubbard model, where each lattice site is occupied by at most two electrons, has been extensively studied in recent years as a model of strongly correlated electron systems 12 But in many systems, such as molecular solids $C_{60}$ and $A_{x} C_{60}$, tion band is degenerate which allows the occupation of more than two electrons per lattice site. For these systems, studying the degenerate Hubbard model on a lattice is the first step toward the understanding of electron-electronic correlations. Yet, there has been little progress since the pioneering works of Chao and Gutzwiller in early 1970s.

Recently, we succeeded in obtaining the Gutzwiller-like analytical solutions to degenerate Hubbard models using a scheme similar to the original Gutzwiller-Approximation. report of our results has been published.6 The solution was used successfully to interpret the unusual metal-insulator transitions observed in fullerides $A_{x} C_{60}$. details of our derivation and the approximation. In addition, effects of the crystal lattice structure on the metal-insulator transition and the effective mass are also investigated.

We consider the $N$-fold degenerate Hubbard model defined on a lattice

$$
H=\sum_{<i j>=n . n ., \alpha} t c_{j, \alpha}^{+} c_{i, \alpha}+\frac{U}{2} \sum_{i, \alpha \neq \beta} n_{i, \alpha} n_{i, \beta},
$$

where $\langle i j\rangle$ are nearest neighbor lattice sites, $\alpha=(r, \sigma)$ includes both the spin $(\sigma=\uparrow, \downarrow)$ and the orbital $(r=1,2, \cdots, N)$ indices, and $n_{i, \alpha}=c_{i, \alpha}^{+} c_{i, \alpha}$ is the number operator. Both the Hubbard $U$ and the nearest neighbor hopping $t$ are assumed to be independent of lattice sites, electron spins, and molecular (atomic) orbitals. Let $L$ be the total number of lattice sites, $x$ the average number of particles per site. For simplicity, only total symmetric states are considered. Thus, the number of electrons per orbital per spin is $m=x L / 2 N$. For an integer $x$ one expects an insulating ground state for $U>>t$. In such an insulating state there are $x$ localized electrons at each lattice site. Hopping between sites are quenched due to the large correlation energy cost. On the other hand, for $U=0$ the system is metallic as electrons hop between lattice sites to lower the kinetic energy. One expects that there exists a critical $U_{c}$ across which the system undergoes a metal-insulator transition. In general, the insulating ground state can be magnetically ordered. We will consider only non-magnetic 
states. Though the qualitative results described below may be valid for magnetically ordered states, it is clear that further works are required to include the magnetic ordering.

If an electron hops from a site with $x$ particles to a neighboring site which also has $x$ particles, then the correlation energy cost, $U\left(C_{x-1}^{2}+C_{x+1}^{2}-2 C_{x}^{2}\right)=U$, is independent of the band filling $x$ and the degeneracy $N$. The gain in kinetic energy is of the order of band width. Thus, in a simple mean field argument one might expect that $U_{c}$ to be independent of the band filling $x$ and the degeneracy $N$. We will show that this is not true. In fact the solution we obtained in a Gutzwiller-type approximation predicts that $U_{c}$ depends sensitively on both $x$ and $N$.

Metal-insulator transition in non-degenerate Hubbard models have been extensively studied in recent years. 1 At the half-filling (one electron per site) it is known: (1) In 1D, the Bethe Ansatz exact solution 8 shows that the ground state is insulating for $U>0$. (2) In 2D, the quantum Monte Carlo calculations suggest an insulating ground state for a moderate $U .8$ In addition, for an extended Hubbard model (the so called Kievelson-SchrifferSu-Heeger model) it has been shown exactly that the Mott-Hubbard transition exists at a non-zero finite $U_{c}$, 2, and the $U_{c}$ agrees with that obtained by the Gutzwiller-Approximation. (3) In $3 \mathrm{D}$ or higher it is generally believed that a finite $U_{c}$ exists. (4) In the infinite dimensions the Gutzwiller-Approximation is exact. From these results we conclude that the Gutzwiller-Approximation provides a reasonable estimate of the Mott-Hubbard transition in 3D degenerate Hubbard models.

In the simple Hubbard model each lattice site are either empty, singly-occupied, or doubly-occupied. Within the Gutzwiller approach, one introduces a wave function which is a linear combination of states with different number of doubly-occupied sites with weighting factors to be determined variationally. To make analytical calculations possible, Gutzwiller made two approximations:5 1) In the thermodynamic limits, the expectation value of an operator in the variational function is approximated by the largest term contributing to it. This approximation is justified by the fact that the weighting factor, which includes various combinatorial factors, is a sharply peaked function. 2) In evaluating the expectation the 
spatial correlations of doubly-occupied sites are neglected. This is equivalent to the mean field approximation. For the $N$-fold degenerate Hubbard model, the problem is more complex as each site can be occupied by 0 to $2 N$ number of electrons. Without further approximation the combinatorial counting becomes intractable. However, close to the metal-insulator transition one expects that large fluctuations from the average occupancy $x$ is small as they cost large correlation energy. This leads us to make the additional approximation: 3) Each lattice site is restricted to three possible states: "empty" (E) - with $x-1$ electrons, "singlyoccupied" $(S)$ - with $x$ electrons, or "doubly-occupied" (D) - with $x+1$ electrons. This approximation makes it possible to do variational calculations following similar steps of the original Gutzwiller-Approximation. We will refer to conditions 1), 2), 3) as the GeneralizedGutzwiller-Approximation (GGA) for the degenerate Hubbard model.

Let $\Gamma, \Omega, \Lambda$ be the total number of sites for types (D), (S), (E) respectively. Approximation 3) and the conservation of particles require that $L=\Gamma+\Omega+\Lambda, \Lambda=\Gamma$. For a fixed $\Gamma$, there are many possible ways of participating $L$ lattice sites. A given (D) site is distinguished by identifying flavors $p_{1}, p_{2}, \ldots, p_{x+1} \in(1,2,3, \ldots, 2 N)$ of all $x+1$ particles on the site. The numbers of lattice sites occupied by the same configuration in general depends on the configuration. For clarity, we will use the short-hand notation $\gamma_{p}\left(p=1,2,3, \ldots, C_{2 N}^{x+1}\right)$ for this number. There are $C_{2 N}^{x+1}=\frac{(2 N) !}{(x+1) !(2 N-x-1) !}$ distinct possible configurations for each (D) sites. Similarly we define $\omega_{r}$ and $\lambda_{q}$ for sites of type (S) and (E). Respectively, there are $C_{2 N}^{x}$ and $C_{2 N}^{x-1}$ possible configurations for these sites. One has

$$
\sum_{p=1}^{C_{2 N}^{x+1}} \gamma_{p}=\Gamma, \quad \sum_{r=1}^{C_{2 N}^{x}} \omega_{r}=\Omega, \quad \sum_{q=1}^{C_{2 N}^{x-1}} \lambda_{q}=\Lambda
$$

Following Gutzwiller 0 , one introduces the weighting factor $0<\eta<1$ for each (D) site, the GGA variational wavefunction $\mid \phi_{G G A}>$ can be written as a linear combination of states with different $\left\{\lambda_{p}\right\},\left\{\omega_{r}\right\},\left\{\lambda_{q}\right\}$,

$$
\left|\psi_{G G A}>=\sum_{\left\{\lambda_{p}\right\},\left\{\omega_{r}\right\},\left\{\lambda_{q}\right\}}(\eta)^{\Gamma} A\left(\left\{\lambda_{p}\right\},\left\{\omega_{r}\right\},\left\{\lambda_{q}\right\}\right)\right| \phi_{\left\{\lambda_{p}\right\},\left\{\omega_{r}\right\},\left\{\lambda_{q}\right\}}>.
$$

The coefficient $A$ is proportional to the total number of possible ways of participating the 
lattice given $\left\{\lambda_{p}\right\},\left\{\omega_{r}\right\},\left\{\lambda_{q}\right\}$,

$$
\begin{aligned}
\left|A\left(\left\{\gamma_{p}\right\},\left\{\omega_{r}\right\},\left\{\lambda_{q}\right\}\right)\right|^{2} & =\text { const } \times \prod_{p=1}^{C_{2 N}^{x+1}} C_{L-\sum_{i<p} \gamma_{i}}^{\gamma_{p}} \times \prod_{r=1}^{C_{2 N}^{x}} C_{L-\Gamma-\sum_{i<r} \omega_{i}}^{\omega_{r}} \times \prod_{q=1}^{C_{2 N}^{x-1}-1} C_{L-\Gamma-\Omega-\sum_{i<q} \lambda_{i}}^{\lambda_{q}} \\
& =\text { const } \frac{L !}{\left(\prod_{p=1}^{C_{2 N}^{x+1}} \gamma_{p} !\right) \times\left(\prod_{r=1}^{C_{2 N}^{x}} \omega_{r} !\right) \times\left(\prod_{q=1}^{C_{2 N}^{x-1}} \lambda_{q} !\right)}
\end{aligned}
$$

where, the const is determined by the normalization condition. Given the general wavefunction Eq.(3), the expectation value of an operator is difficult to calculate. Within the Gutzwiller-Approximation one assumes that in the thermodynamical limit only the largest term contributes significantly to the expectation. For such an optimal term, the symmetry of the problem dictates that

$$
\begin{aligned}
& \gamma_{p}=\gamma=\Gamma / C_{2 N}^{x+1}, \\
& \lambda_{q}=\lambda=\Lambda / C_{2 N}^{x-1}=\Gamma / C_{2 N}^{x-1}, \\
& \omega_{r}=\omega=\Omega / C_{2 N}^{x}=(L-2 \Gamma) / C_{2 N}^{x} .
\end{aligned}
$$

The relationship between $\eta$ and $\Gamma$ is determined by the largest term in $\left\langle\psi_{G G A} \mid \psi_{G G A}\right\rangle$. This leads to

$$
\begin{array}{r}
\eta^{2(\Gamma+1)}|A(\gamma+1, \gamma, \gamma, \cdots, \gamma ; \omega-1, \omega-1, \omega, \cdots, \omega ; \lambda+1, \lambda, \lambda, \cdots, \lambda)|^{2} \\
=\eta^{2 \Gamma}|A(\gamma, \gamma, \gamma, \cdots, \gamma ; \omega, \omega, \omega, \cdots, \omega ; \lambda, \lambda, \lambda, \cdots, \lambda)|^{2} .
\end{array}
$$

Using Eq.(2), (4) and (5) one obtains

$$
\eta=\frac{\sqrt{\gamma \lambda}}{\omega}=\frac{\Gamma}{L-2 \Gamma} \sqrt{\frac{(x+1)(2 N-x+1)}{x(2 N-x)}} .
$$

Keeping only the largest term in the normalization condition $\left\langle\psi_{G G A}\right| \psi_{G G A}>=1$ one finds

$$
(\text { const })^{-2}=\eta^{2 \Gamma}|A(\gamma, \gamma, \gamma, \cdots, \gamma ; \omega, \omega, \omega, \cdots, \omega ; \lambda, \lambda, \lambda, \cdots, \lambda)|^{2} .
$$

The optimal value of $\Gamma$ (or $\eta$ ) is determined variationally by minimizing the total energy. The energy consists of the kinetic and the correlation part. The kinetic energy is calculated by evaluating the hopping term in Eq.(1). This requires the calculation of correlation functions 


$$
\rho(i, \alpha ; j, \alpha)=<\psi_{G G A}\left|c_{i, \alpha}^{\dagger} c_{j, \alpha}\right| \psi_{G G A}>
$$

Because each site can be occupied by $x+1, x$, or $x-1$ electrons only, the case $i=j$ is easily evaluated to give

$$
\rho(i, \alpha ; i, \alpha)=\frac{\gamma C_{2 N-1}^{x}+\omega C_{2 N-1}^{x-1}+\lambda C_{2 N-1}^{x-2}}{L}=\frac{x}{2 N}
$$

Where, in the last step Eq.(5) is used. For the case $i \neq j$ there are three distinct possibilities: (a) None of two sites are doubly-occupied; (b) One of two is doubly-occupied; (c) Both are doubly-occupied. Summing all cases together leads to

$$
\begin{aligned}
\rho(i, \alpha ; j \neq i, \alpha) & =\frac{\omega \lambda C_{2 N-1}^{x-1} C_{2 N-1}^{x-1}+2 \eta \omega^{2} C_{2 N-1}^{x-1} C_{2 N-1}^{x}+\omega^{3} \lambda^{-1} C_{2 N-1}^{x} C_{2 N-1}^{x}}{L(L-m)} \\
& =\frac{\Gamma(L-2 \Gamma) x(2 N-x+1)}{2 N L^{2}(2 N-x)}\left(1+\eta \frac{\omega C_{2 N-1}^{x}}{\lambda C_{2 N-1} x-1}\right)^{2} \\
& =\frac{\Gamma(L-2 \Gamma)}{2 N L^{2}(2 N-x)}(\sqrt{x(2 N-x+1)}+\sqrt{(x+1)(2 N-x)})^{2} .
\end{aligned}
$$

In deriving these results Eq.(5) and (7) are used. The kinetic energy per particle can be written as

$$
K . E .=Q(x, \Gamma, N) \bar{\epsilon}(x)
$$

where

$$
\bar{\epsilon}(x)=\frac{2}{x L} \sum_{\mathbf{k}<\mathbf{k}_{\mathbf{F}}} \epsilon(\mathbf{k}) \leq 0
$$

is the average kinetic energy per particle in the absence of the correlation. (The bare orbital energy has been chosen to be zero.) The quotient $Q(x, \Gamma, N)$, 哥 which represents the reduction in kinetic energy of the correlated system in comparison with that of the non-correlated system, is given by

$$
\begin{aligned}
Q(x, \Gamma, N) & =\frac{\rho(i, \alpha ; j \neq i, \alpha)}{\rho(i, \alpha ; i, \alpha)} \\
& =\frac{\Gamma(L-2 \Gamma)}{x L^{2}(2 N-x)}(\sqrt{x(2 N-x+1)}+\sqrt{(x+1)(2 N-x)})^{2} .
\end{aligned}
$$

The correlation energy is determined by the expectation 


$$
\begin{aligned}
\sum_{i, \alpha \neq \beta}<\psi_{G G A}\left|n_{i, \alpha}^{\dagger} n_{i, \beta}\right| \psi_{G G A}> & =\Gamma C_{x+1}^{2}+\Omega C_{x}^{2}+\Lambda C_{x-1}^{2} \\
& =L \frac{x(x-1)}{2}+\Gamma
\end{aligned}
$$

The same expectation when evaluated in the paramagnetic insulating state, where there are $x$ electrons localized at each site, gives a value $L \frac{x(x-1)}{2}$. The difference, $\Gamma$, is the increase in the correlation energy in the metallic state. As the kinetic energy in the paramagnetic insulating state is zero, the increase in total energy per particle in the metallic state is,

$$
E(N, x)=Q(x, \Gamma, N) \bar{\epsilon}(x)+\frac{\Gamma}{x L} U
$$

Minimizing this energy with respect to $\Gamma$, one obtains the fraction of doubly-occupied sites,

$$
\frac{\Gamma}{L}=\frac{1}{4}\left(1-\frac{U}{U_{c}}\right)
$$

and the total energy per particle

$$
E(x, N)=-\frac{U_{c}(x, N)}{8 x}\left(1-\frac{U}{U_{c}(x, N)}\right)^{2} .
$$

Where, the critical $U$ is given by

$$
U_{c}(N, x)=\frac{(\sqrt{x(2 N-x+1)}+\sqrt{(x+1)(2 N-x)})^{2}}{2 N-x}|\bar{\epsilon}(x)| .
$$

As $U$ increases toward $U_{c}$, the number of doubly-occupied sites approaches zero. For $U>U_{c}$ the paramagnetic insulating state has a lower energy than the metallic state. Thus the GGA predicts a first order Mott-Hubbard metal-insulator transition at $U_{c}$.

Eq.(19) is our main result. It shows that $U_{c}$ depends sensitively on both the number of electrons per lattice site and the band degeneracy. In Fig.1 we show several examples of this dependence with the band energy calculated from the nearest neighbor tight-binding Hamiltonian on the simple cubic lattice. A prominent feature is that $U_{c}$ is the largest at the half filling, $U_{c}(N, N)=4(N+1) \bar{\epsilon}(N)$. This implies that if the system is insulating at the half filling, then for all other fillings with integer number of electron per site the system is also insulating. This result has been used successfully to explain the unusual metallic/insulating properties of fulleride materials $A_{x} C_{60}$. 
The critical correlation also depends sensitively on the lattice structure through the band energy $\bar{\epsilon}(x)$. In Fig.2 we show examples of the phase diagram with $\bar{\epsilon}(x)$ calculated from the 2-dimensional square, the simple-cubic, and the body-center-cubic lattice. As the number of nearest neighbor increases $U_{c}$ decreases with respect to the band width.

Our result, Eq.(19), reproduces all previously known analytical Gutzwiller solutions. The three cases that we are aware of are: (1) $N=1$, in this case the only commensurate filling is $x=1$, Eq.(19) leads to the well known Brinkman-Rice criteria $U_{c}=8|\bar{\epsilon}|$ 目 $(2)$ $N=2$ and $x=1$, this is the case studied by Chao and Gutzwiller in early 1970s. The critical correlation they obtained, $U_{c}=\frac{10+4 \sqrt{6}}{3}|\bar{\epsilon}|$, is identical to that given by Eq.(19). (3) $N \rightarrow \infty$ with a finite $x$, in this case the fermion problem is equivalent to the boson problem. The boson Hubbard model has been widely studied in recent years $10-12$. Our result, $U_{c}=(\sqrt{x}+\sqrt{x+1})^{2}|\bar{\epsilon}(x)|$, is the same as that obtained for the boson Hubbard model using the Gutzwiller-Approximation.11 It is also close to the value given by quantum Monte Carlo calculations. 12 This suggest that in the limit of high band degeneracy our Generalized-Gutzwiller-Approximation is quite accurate. It would be very interesting to do Monte Carlo calculations explicitly for the degenerate model and compare with our results.

The physical properties of the system can undergoes dramatic changes when the metalinsulator transition is approached. These include the magnetic susceptibility and the transport effective mass. As Brinkman-Rice first pointed out, 0 the effective mass $m^{*}$ is critically enhanced near the metal-insulator transition. The enhancement is proportional to $1 / Q$. Using Eq.(14) and (17) one finds

$$
\frac{m^{*}}{m_{b}}=\frac{1}{Q}=\frac{8|\epsilon(x)|}{x U_{c}(x, N)\left(1-\left(U / U_{c}\right)^{2}\right)},
$$

where $m_{b}$ is the effective band mass. One observes that the effective mass diverges quadratically as the metal-insulator transition is approached. However, the enhancement is less dramatic for large $N$ or large $x$. Brinkman-Rice also showed that the magnetic susceptibility also diverges as $1 /\left(1-\left(U / U_{c}\right)^{2}\right)$. We suspect that similar result holds for the degenerate Hubbard model. However, this remains to be proven as our calculation is valid only for 
non-magnetic states.

In conclusion we present results of analytical studies on degenerate Hubbard models using the Generalized-Gutzwiller-Approximation. It is shown that for any filling with integer electron per lattice site there exists a critical correlation energy above which the system is a Mott-Hubbard insulator. The general expression we found for $U_{c}$ depends sensitively on the band degeneracy, the number of electron per site and the lattice structure. It also reproduces all previous know Gutzwiller solutions as special limits.

\section{ACKNOWLEDGMENTS}

This work is supported by the Petroleum Research Fund and the U.S. Department of Energy. 


\section{REFERENCES}

${ }^{1}$ J. Hubbard, Proc. Roy. Soc. A276, 236 (1963). ibid. A277, 237 (1964). ibid. A281, 401 (1964).

${ }^{2}$ A. Montorsi and M. Rasetti, in The Hubbard Model - Recent Results, edited by M. Rasetti, World Scientific (Singapore) 1991.

${ }^{3}$ For reviews on solid fullerenes and fullerides see Physics and Chemistry of Fullerenes, ed. P. W. Stephens, World Scientific (1993); Solid State Physics Vol. 48, ed. H. Ehrenreich and F. Spaepen, Academic Press (1994).

${ }^{4}$ K. A. Chao and M. C. Gutzwiller, J. Appl. Phys. 42, 1420 (1971). K. A. Chao, Phys. Rev. B 4, 4034. J. Phys. C 7, 127 (1974).

${ }^{5}$ M. C. Gutzwiller, et al., Phys. Rev. 137, A1726 (1965). W. Brinkman and T. Rice, Phys. Rev. B 2, 4302 (1970).

${ }^{6}$ J. P. Lu, Phys. Rev. B 49, 5687 (1994).

${ }^{7}$ R. Mota et al., Int. Jour. Quan. Chem., to appear.

${ }^{8}$ E. H. Lie and F. Y. Wu, Phys. Rev. Lett. 20, 1445 (1968).

${ }^{9}$ D. J. Scalapino, in High Temperature Superconductivity Proceedings, edited by K.S. Bedell et al., Addison-Wesley (1990).

${ }^{10}$ M. P. A. Fisher et al., Phys. Rev. B 40, 546 (1989). K. Sheshdari et al., Europhys. Lett. 22, 257 (1993).

${ }^{11}$ D. S. Rokhsar and B. G. Kotliar, Phys. Rev. B 44, 10328 (1991). W. Kruth et al., Phys. Rev. B 45, 3137 (1992).

${ }^{12}$ W. Kruth et al., Phys. Rev. Lett. 67, 2037 (1991). R. T. Scalettar et al., Phys. Rev. Lett. 66, 3144 (1991). 


\section{FIGURES}

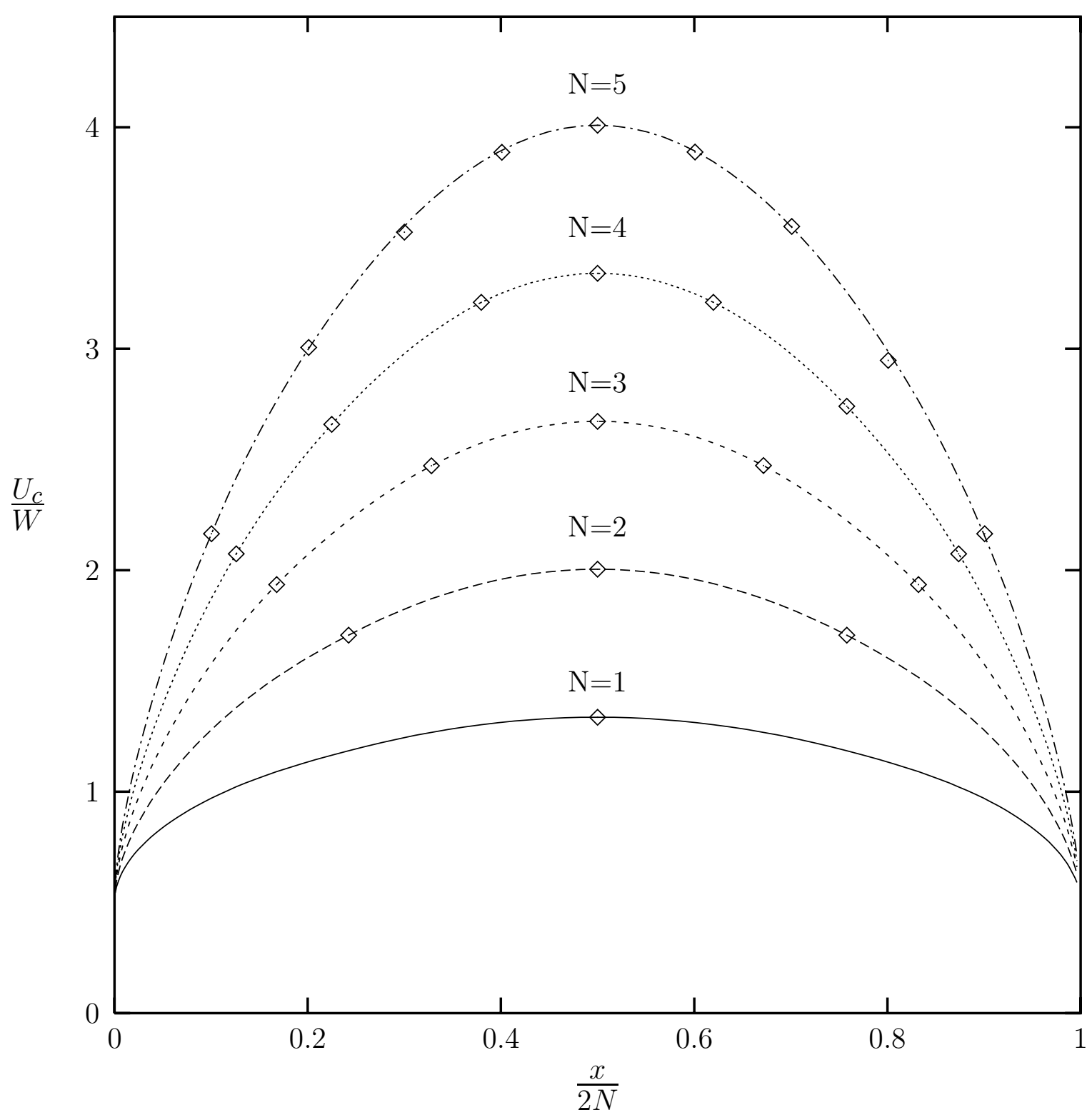

FIG. 1. Phase diagrams of metal-insulator transitions in degenerate Hubbard models as predicted by Eq.(19). Critical correlation $U_{c}$ (scaled by the band width) is plotted against the band filling $x$ (scaled by $2 N$ ). The band energy is calculated from the n.n. tight-binding model on the simple-cubic lattice. Shown are results for $N=1,2,3,4,5$. The $U_{c}$ is the largest at the half-filling $x=N$. In both Fig.1 and Fig.2 only points, corresponding to integer number of electrons per site, are meaningful. Lines are draw using Eq.(19). 


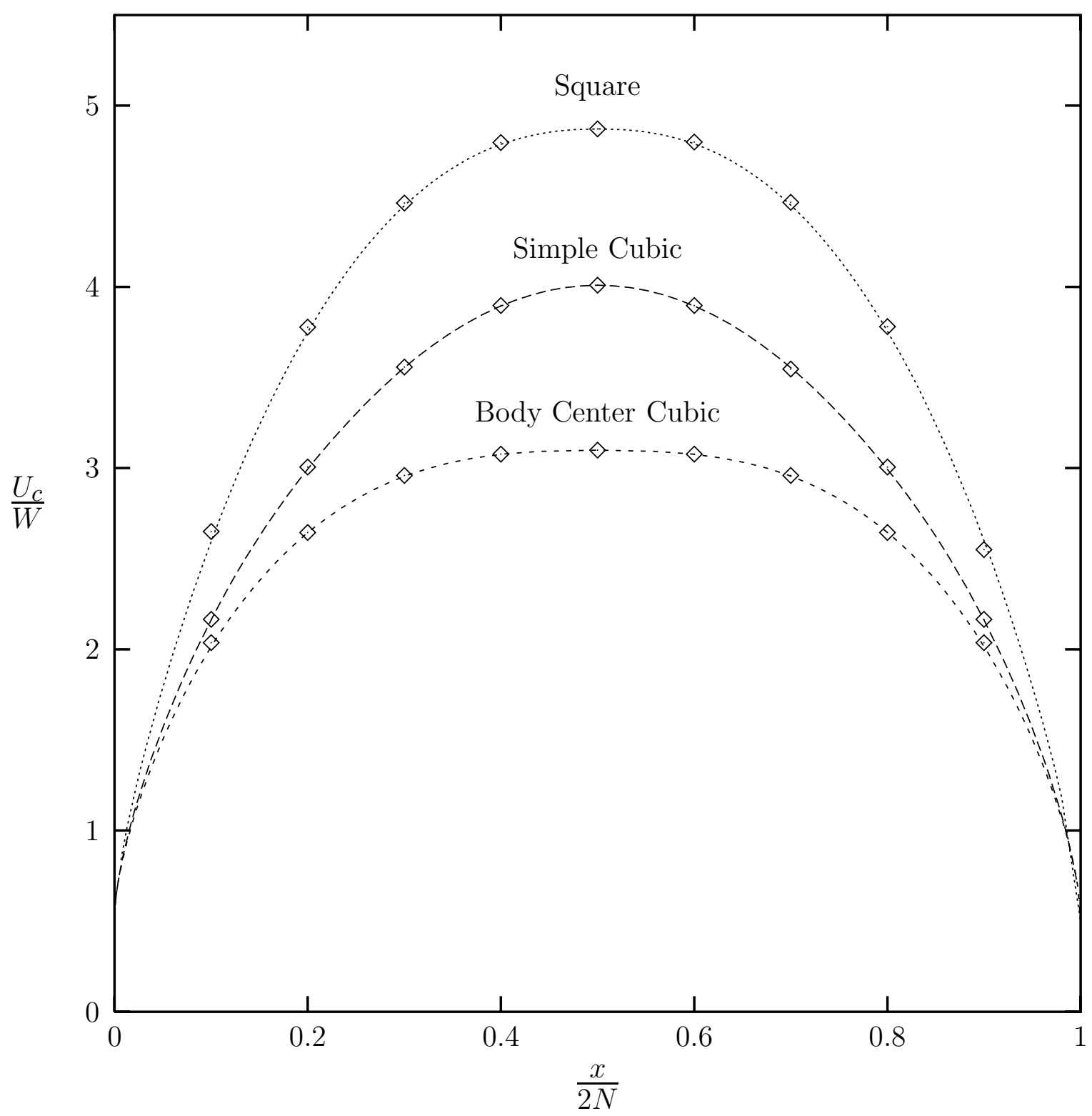

FIG. 2. Phase diagrams of metal-insulator transitions in degenerate Hubbard models for different lattice structures. Shown are three examples with $N=5$ and the band structure calculated for the 2-D square, 3-D simple-cubic, and 3-D body-center-cubic lattices with the nearest neighbor tight-binding hamiltonian. Note that given the same band filling and the degeneracy, the critical correlation can changes substantially from one type of lattice structure to another. 